\title{
Domain wall pinning in notched nanowires
}

\author{
H. Y. Yuan and X. R. Wang* \\ Physics Department, The Hong Kong University of Science and Technology, Clear Water Bay, Kowloon, Hong Kong \\ and HKUST Shenzhen Research Institute, Shenzhen 518057, China
}

(Received 8 November 2013; revised manuscript received 20 January 2014; published 20 February 2014)

\begin{abstract}
We theoretically and numerically study magnetic domain wall (DW) pinning at a notch in a magnetic nanowire. Based on the static DW equation, a general relationship between an external field and a DW structure for a given notch geometry is found. By estimating the field below which this relationship holds, we obtain the depinning field theoretically. Our theoretical estimate of the depinning field compares well with simulation results. Furthermore, our theory explains well why the depinning field of a transverse wall of one chirality is larger than that of the opposite chirality.
\end{abstract}

DOI: 10.1103/PhysRevB.89.054423

PACS number(s): 75.60.Ch, 75.70.Ak

\section{INTRODUCTION}

The motion of domain walls (DWs) in magnetic nanowires has attracted much attention in recent years due to its potential applications in magnetic memory devices [1] and magnetic logic gates [2]. These applications are likely to use pinning effects of a notch on an otherwise homogeneous nanowire to manipulate DW positions. Although much knowledge of DW pinning in a notched wire has been obtained from experiments and micromagnetic simulations [3-23], our current understanding of DW pinning and depinning is quite limited. It is numerical [18] and experimental $[3,10,12,15]$ fact that thickness inhomogenities [3] and geometrical constrictions $[10,12,15]$ can pin a DW. The depinning field depends on the geometry of the constrictions. It is also known [18] that depinning fields depend on the depinning process: the static depinning field is larger than the dynamic depinning field for a notched nanowire. One popular view $[12,15,24]$ is to assume pinning as a trap potential well for DWs. However, the collective model, the base of the concept of the trap potential, may not be stable against spin-wave emission [25,26]. In general, a DW, whose structure is substantially deformed near a notch, is by no means a point particle, and it cannot be described by one coordinate like a DW center. Even worse, a DW can only stay at most at a few special locations on a notched wire. Thus, a trap potential as a function of one variable like the DW center is ill defined. A seminal analytical work by Bruno [4] suggests that the pinning can only occur at narrower parts of a constrained nanowire. Thus, it cannot explain DW pinning by an antinotch [18]. In summary, a proper concept and a unified analytical theory for DW pinning by notches are lacking, and that is the focus of the current work.

In this paper, a relationship between an external field and a static DW structure is obtained. By estimating the field below which the relationship holds, we obtain the depinning field that compares well with simulation results. The paper is organized as follows. Our model, theory, and the depinning field formula are in Sec. II. A comparison of the theory with simulation results is presented in Sec. III, followed by the conclusion.

\footnotetext{
*Corresponding author: phxwan@ust.hk
}

\section{MODEL AND THEORETICAL APPROACH}

We consider a magnetic nanowire of length $l$, width $w$, and thickness $t$ with a notch on the top edge. The $z$ axis is along the wire, the $y$ axis is along the width direction, and the $x$ axis is in the thickness direction. A side view of a nanowire with a rectangular notch that is $d$ wide and $w_{1}$ deep is shown in Fig. 1.

The magnetic wire is described by the following free energy:

$$
\begin{aligned}
E(\mathbf{m})= & \int\left[\frac{A}{\mu_{0} M_{s}^{2}}\left(\nabla m_{i}\right) \cdot\left(\nabla m_{i}\right)+f_{a n}(\mathbf{m})+f_{d}(\mathbf{m})\right. \\
& \left.+f_{0}(\mathbf{m})\right] d V,
\end{aligned}
$$

where $\mathbf{m}$ is the unit vector of magnetization, $A$ is the exchange constant, $M_{s}$ is saturation magnetization, $f_{a n}$ is anisotropy energy density, $f_{d}=-1 / 2 \mathbf{m} \cdot \mathbf{h}_{d}$ is magnetostatic energy density, and $f_{0}=-\mathbf{m} \cdot \mathbf{h}$ is the Zeeman energy density; here $f_{d}, f_{a n}$, and $f_{0}$ are in units of $\mu_{0} M_{s}^{2}$, and magnetostatic field $\mathbf{h}_{d}$ and external field $\mathbf{h}$ are in units of $M_{s}$. The summation convention is applied for the double appearance of index $i$. Minimizing this energy yields the Euler-Lagrange equation,

$$
-l_{e x}^{2} \nabla^{2} m_{i}+\frac{\partial f_{o t}}{\partial m_{i}}-h_{d, i}+2 \lambda m_{i}=0,
$$

where $i=1,2,3, f_{o t}=f_{a n}+f_{0}, l_{e x} \equiv \sqrt{2 A / \mu_{0} M_{s}^{2}}$ is the exchange length [27] and $\lambda$ is the Lagrange multiplier due to the constraint $\mathbf{m} \cdot \mathbf{m}=1$. Equation (2) is equivalent to $\mathbf{m} \times \mathbf{h}_{\text {eff }}=0$, where the effective field is defined as

$$
h_{\mathrm{eff}, i} \equiv l_{e x}^{2} \nabla^{2} m_{i}-\frac{\partial f_{o t}}{\partial m_{i}}+h_{d, i},
$$

where $i=1,2,3$. This means that the magnetization of a static DW is parallel or antiparallel to the effective field everywhere. If Eq. (2) has a DW solution of a boundary condition $\partial \mathbf{m} / \partial \mathbf{n}=0$, where $\mathbf{n}$ is the normal direction of the wire boundary, it means that a DW is pinned [4,28]. Mathematically, the depinning field is the minimal external field at which Eq. (2) does not have a DW solution. In early publications [29], it was shown that Eq. (2) does not have a DW solution for a homogeneous nanowire in the presence of an external field along the wire. For a wire with a notch, the 


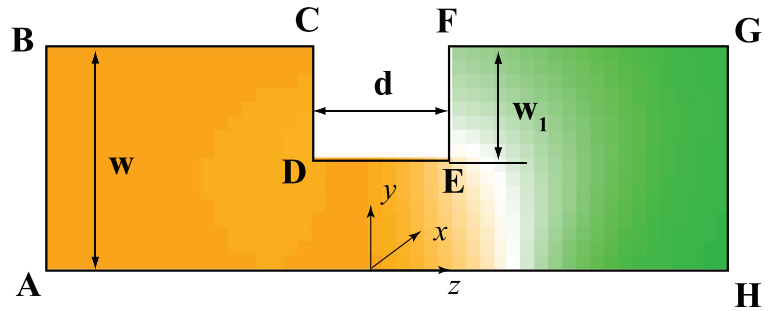

FIG. 1. (Color online) Side view of a magnetic nanowire with dimensions $l \times w \times t$. The dimensions of a rectangular notch are $d \times w_{1} \times t$. A DW is pinned at the right-hand side of the notch. Color varying from orange to green represents the values of $m_{z}$ (orange for $m_{z}=1$ and green for $m_{z}=-1$ ). White indicates the DW center, where $m_{z}=0$.

criterion for the existence of a DW solution of Eq. (2) is not yet known. To find such a criterion, we shall first find identities hidden in Eq. (2). Then, by estimating the possible breakdown of the identity, we obtain the approximate depinning field.

Multiplying Eq. (2) with $\partial m_{i} / \partial x_{j}$ and summing over $i=$ $1,2,3$, one has

$$
-l_{e x}^{2} \frac{\partial m_{i}}{\partial x_{j}} \nabla^{2} m_{i}+\frac{\partial f_{o t}}{\partial x_{j}}-h_{d, i} \frac{\partial m_{i}}{\partial x_{j}}+2 \lambda m_{i} \frac{\partial m_{i}}{\partial x_{j}}=0,
$$

where $j=1,2,3$. Using identities

$$
\frac{\partial g}{\partial x_{j}} \nabla^{2} g=\nabla \cdot\left(\frac{\partial g}{\partial x_{j}} \nabla g\right)-\frac{1}{2} \frac{\partial}{\partial x_{j}}(\nabla g)^{2}
$$

and $\mathbf{m} \cdot \partial \mathbf{m} / \partial x_{j}=0$ from $\mathbf{m} \cdot \mathbf{m}=1$, we have

$$
l_{e x}^{2} \nabla \cdot\left(\frac{\partial m_{i}}{\partial x_{j}} \nabla m_{i}\right)=\frac{\partial \rho_{1}}{\partial x_{j}}-h_{d, i} \frac{\partial m_{i}}{\partial x_{j}},
$$

where $\rho_{1}=\frac{1}{2} l_{e x}^{2}\left(\nabla m_{i}\right) \cdot\left(\nabla m_{i}\right)+f_{o t}$ is the total energy density excluding magnetostatic energy density. Integrating this equation over sample $\Omega$ between planes $A B$ and $H G$ and using the Gauss theorem and boundary condition $\partial \mathbf{m} / \partial \mathbf{n}=0$, one obtains

$$
\int_{\partial \Omega} \rho_{1} d \mathbf{S}-\int_{\Omega} \mathbf{h}_{d} \cdot \nabla \mathbf{m} d V=0 .
$$

Here planes $A B$ and $H G$ are inside two domains and are far from the DW that is near the notch. $\partial \Omega$ is the boundary of $\Omega$. This equation is a general result valid for all DWs and notches. For simplicity we restrict our discussion below to the head-to-head transverse wall in a thin nanowire with a rectangular notch, as shown in Fig. 1. As the nanowire thickness is very small, it can be reasonably assumed that the magnetization does not change in the thickness direction. Then the $z$ component of the above equation can be greatly reduced to

$$
t \int_{C D} \rho_{1} d y-t \int_{E F} \rho_{1} d y-\int_{\Omega} \mathbf{h}_{d} \cdot \frac{\partial \mathbf{m}}{\partial z} d V=-2 h t w .
$$

Then, the external field can be expressed as a functional of DW structure,

$$
h=\frac{t \int_{E F} \rho_{2} d y-t \int_{C D} \rho_{2} d y+\int \mathbf{h}_{d} \cdot \frac{\partial \mathbf{m}}{\partial z} d V}{t w_{1}\left(2 w / w_{1}+\bar{m}_{z E F}-\bar{m}_{z C D}\right)},
$$

where $\rho_{2}$ includes only the exchange energy and the anisotropy energy and $\bar{m}_{z E F}$ and $\bar{m}_{z C D}$ are the averaged $z$ components of magnetization on surfaces $E F$ and $C D$, respectively. If a geometrically pinned DW exists, the corresponding DW structure and the external field have to satisfy this identity. Because a DW structure is the balance between its exchange energy and its anisotropy energy, the abrupt change of spin orientation is thereby forbidden, and the right-hand side of Eq. (8) should be bounded. If we keep increasing the external field, Eq. (8) will break down above a critical field. The breakdown of Eq. (8) means that the DW is moved out of the notch and the critical field corresponds to the depinning field. What remains is to estimate this critical field.

Before moving forward, let us first identify the meaning of all terms in Eq. (8). The first two terms in the numerator are the exchange and anisotropy energy difference of surface $E F$ and

(a)

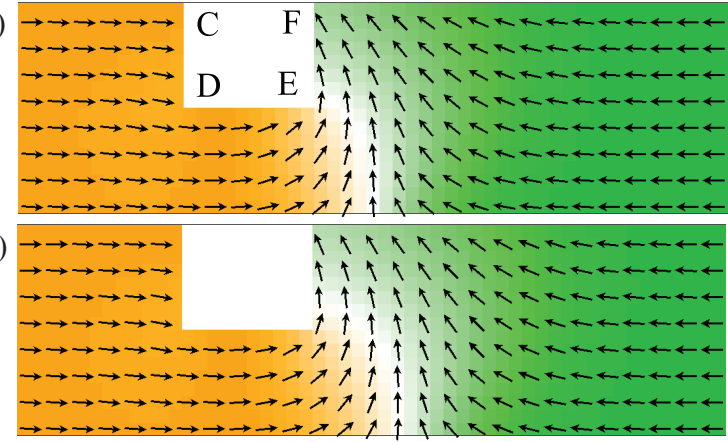

(c)

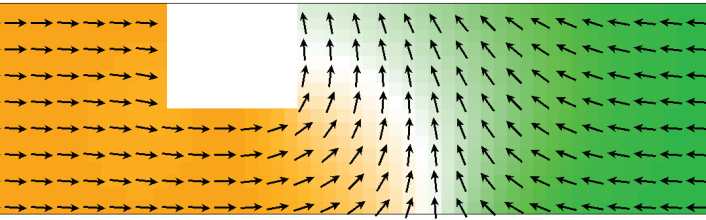

(d)

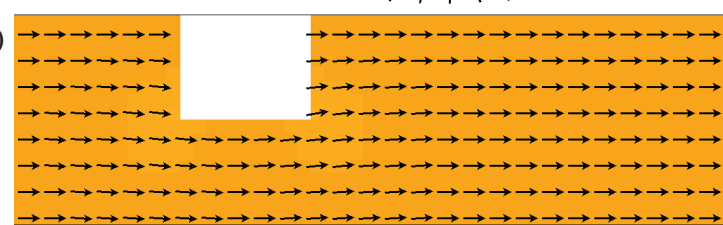

(e)

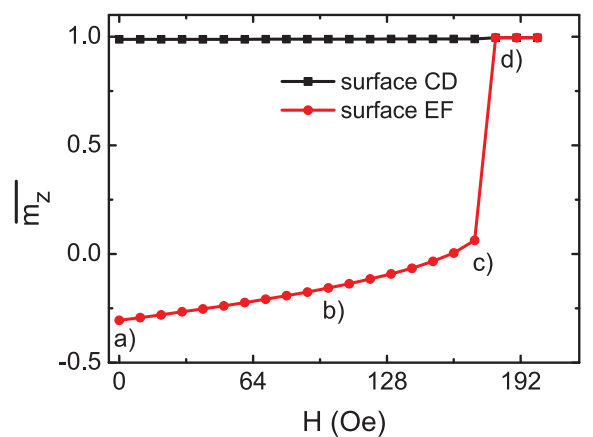

FIG. 2. (Color online) Snapshots of the spin configuration around the notch as the field increases from zero up over the depinning field. (a) $H=0 \mathrm{Oe}$, (b) $H=100 \mathrm{Oe}$, (c) $H=170 \mathrm{Oe}$, and (d) $H=180$ Oe. For clarity, each spin represents the average magnetization of four $4 \times 4 \times 4 \mathrm{~nm}$ cells. The nanowire dimensions are $1000 \times 64 \times 4 \mathrm{~nm}$, and the notch dimensions are $40 \times 32 \times$ $4 \mathrm{~nm}$. (e) The evolution of $\bar{m}_{z C D}$ and $\bar{m}_{z E F}$ with applied field. 
surface $C D$. Suppose a positive field is applied along the $+z$ direction; the DW center will be shifted along the $+z$ direction $[6,7]$. Figures $2(a)-2(d)$ show the change of spin configuration as the external field increases from zero to the depinning field. Specifically, Fig. 2(a) is the initial configuration at zero field where the spins on $E F$ tilt at an angle away from the $E F$ surface. As field increases, these spins rotate clockwise toward the $E F$ surface [Fig. 2(b)]. Right before the DW is moved out of the notch, the spins align almost parallel to the $E F$ plane, as Fig. 2(c) shows. After the field exceeds a critical value, the DW center line near $E F$ begins to deviate from the notch, and the DW is removed from the notch. Spins in equilibrium states are normal to the $E F$ surface [Fig. 2(d)]. Throughout this process, spins on $C D$ gradually align with the normal direction. The changes of $\bar{m}_{z E F}$ and $\bar{m}_{z C D}$ as a function of external field are shown in Fig. 2(e), which clearly shows that the state at the largest field at which the DW is marginally pinned appears to have $\bar{m}_{z E F}=0$ and $\bar{m}_{z C D}=1$. We estimate $T_{1}$ for the critical state as

$$
\begin{aligned}
T_{1} & \equiv t \int_{E F} \rho_{2} d y-t \int_{C D} \rho_{2} d y \\
& \approx \frac{1}{2} t w_{1}\left(\frac{l_{e x}}{\Delta}\right)^{2}+t w_{1} \frac{K_{a n}}{\mu_{0} M_{s}^{2}},
\end{aligned}
$$

where $\Delta$ is the DW width around the notch and $K_{a n}>0$ is the crystalline anisotropy along the $z$ direction. The third term in the numerator is the magnetostatic interaction. In a domain region, the magnetization is homogeneous, i.e., $\partial \mathbf{m} / \partial z \approx 0$. Thus, the contribution of the domain to this term is zero. In a DW region, only surface charges are taken into account because volume charges contribute little in comparison with surface charges [30-32]. The magnetostatic field can thereby be approximated as an effective anisotropy field [30,31]: $\mathbf{h}_{d} \approx-K_{y} m_{y} e_{y}-K_{z} m_{z} e_{z}$. Here $K_{y}=K_{y}(w, t)$ comes from the contribution of surface charges on the top surface $y=w$ and the bottom surface $y=0 ; K_{z}=K_{z}(w, t)$ is from the charges on $E F$. For the critical magnetization distribution where $\bar{m}_{z E F}=0, K_{z}=0$, only $K_{y}$ contributes to the integral. Hence the term can be reduced to

$$
T_{2} \equiv \int \mathbf{h}_{d} \cdot \frac{\partial \mathbf{m}}{\partial z} d V \approx \frac{K_{y}}{\mu_{0} M_{s}^{2}} w_{1} t
$$

It should be stressed that $T_{2}$ is mainly from the deformed DW structure near the notch. For a symmetric DW, this term is zero because the integral on the right-hand side of the DW cancels that on the left-hand side. We can then reasonably expect that the maximum $T_{2}$ appears for a DW with a half structure. That is just the critical state $\bar{m}_{z E F}=0$ discussed earlier.

From the above discussion, the depinning field can be estimated as

$$
h_{c} \approx \frac{1}{2 w / w_{1}-1}\left[\frac{1}{2}\left(\frac{l_{e x}}{\Delta}\right)^{2}+\frac{K_{a n}}{\mu_{0} M_{s}^{2}}+\frac{K_{y}}{\mu_{0} M_{s}^{2}}\right] .
$$

In terms of $T_{1}$ and $T_{2}$, the depinning field is then

$$
h_{c} \approx \frac{T_{1} /\left(w_{1} t\right)+T_{2} /\left(w_{1} t\right)}{2 w / w_{1}-1} .
$$

This is our key prediction of the depinning field of a head-tohead transverse wall depinning field in a rectangular-notched nanowire. Equation (6) is exact, while Eq. (12) is based on the following assumptions: (1) The wire thickness is thin and constant, so that the magnetization is homogeneous in the thickness direction. That is the reason why the simplified relation (12) is thickness independent. For a nanowire thickness around the exchange length, this assumption is almost strictly satisfied [33]. In a real system, wire thickness should be an important parameter for the depinning field. As thickness increases, complex structure like vortex may appear in the system [27], and the depinning mechanism also becomes complex. Moreover, the thickness inhomogeneity in real materials may induce an additional coercive field [3,6]. (2) The volume charges' contribution to the magnetostatic field can be ignored. For a transverse wall where all the spins lie in a plane, this approximation is acceptable [31]. One may still question the validity of these assumptions, and its accuracy should be tested against the simulations presented below.

\section{RESULTS AND DISCUSSION}

We would like to compare our theoretical prediction with simulation results and discuss the validity of our approach. In the simulations, all nanowires are $1000 \mathrm{~nm}$ long and $4 \mathrm{~nm}$ thick. The wire width varies from 40 to $112 \mathrm{~nm}$ as needed. A rectangular notch is located in the nanowire center. Notch width $d$ is $40 \mathrm{~nm}$, and notch depth $w_{1}$ varies. The sample is divided into cubic cells of $4 \times 4 \times 4 \mathrm{~nm}$. The magnetization dynamics is simulated by the OOMMF package [34] with $A=13 \times 10^{-12} \mathrm{~J} / \mathrm{m}, M_{s}=8 \times 10^{5} \mathrm{~A} / \mathrm{m}$, and $K_{a n}=0.5 \times$ $10^{3} \mathrm{~J} / \mathrm{m}^{3}$. The damping constant is $\alpha=0.1$ to accelerate the simulation. The value of $\alpha$ does not affect conclusions since only static DW structures are considered. The initial state is a head-to-head transverse wall of anticlockwise chirality $[11,23]$ pinned in the right-hand side of $E F$ at zero external field, as shown in Fig. 2(a). This state can be generated either by a pad connected at the right end of the nanowire or around the curved corner in a curved nanowire in experiments $[13,35]$. In order to determine the depinning field, an external field is applied along the $+z$ direction and increases at a step of 5 Oe until the DW moves completely out of the notch. The minimum field needed to depin the DW is the depinning field $h_{c}$. We study the depinning field as a function of wire width at a fixed aspect ratio (the ratio of notch depth and wire width) and as a function of notch depth for a fixed wire width.

Figure 3 shows the depinning field as a function of wire width $w$ when the aspect ratio is fixed at one half $\left(w_{1} / w=\right.$ $1 / 2$ ). The simulation shows that depinning field (black crosses) decreases as the nanowire width increases. This relation can be understood from our theory. As the wire width increases, the magnetostatic field from the surface charges becomes weak; $T_{2} /\left(w_{1} t\right)$ thereby decreases. $T_{1} /\left(w_{1} t\right)$ related to the exchange energy difference also decreases because the DW width becomes larger as the nanowire becomes wider [16,27]. As the denominator does not change, the decrease of $T_{2} /\left(w_{1} t\right)$ and $T_{1} /\left(w_{1} t\right)$ leads to the decrease of the depinning field, as can be seen from Eq. (12). The deviation may come from the error in estimation of $T_{2} /\left(w_{1} t\right)$, which relates to magnetostatic energy. The effective $K_{y}$ is calculated for a perfect transverse 


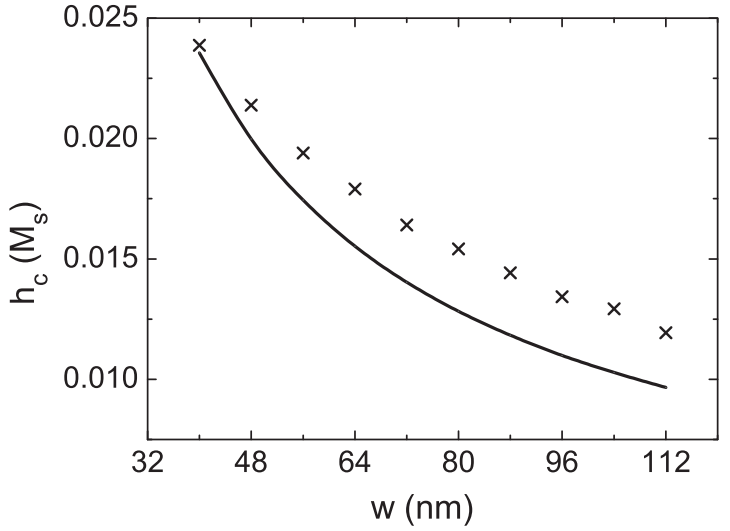

FIG. 3. The notch depth dependence of the depinning field $\left(h_{c}\right)$ of a head-to-head transverse DW of anticlockwise chirality in nanowires with rectangular notches whose width is half of the wire width. The depinning field $h_{c}$ is measured in units of $M_{s}$. Here the black crosses and the black curve are the simulation data and the theoretical estimate, respectively.

wall in a uniform wire [30,31], while a real DW is substantially deformed near a notch. The deformation is more severe for larger $w_{1}$; this is presumably why the discrepancy between numerical results and theoretical estimates is bigger for larger $w_{1}$, as shown in Fig. 3. Im et al. experimentally explored [16] the nanowire width dependence of the depinning field when the aspect ratio is set to about $50 \%$. They also found a similar wire width dependence of the depinning field.

Figure 4 shows the notch depth $\left(w_{1}\right)$ dependence of the depinning field at fixed $w=64 \mathrm{~nm}$. The numerical results (black crosses) show that the deeper the notch is, the larger the depinning field will be. As the wire width is fixed, neither $T_{1} /\left(w_{1} t\right)$ nor $T_{2} /\left(w_{1} t\right)$ changes much. The leading contribution to the depinning field is the denominator, and it leads to an increase of the depinning fields as the notch depth increases. Results in two extremes can be justified. One has

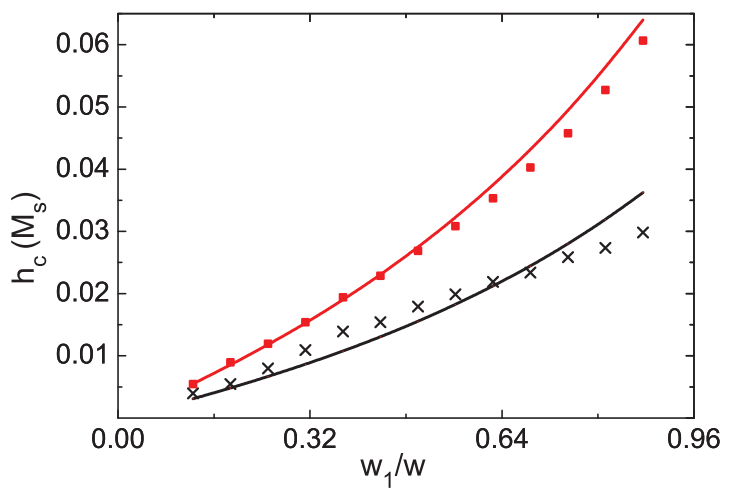

FIG. 4. (Color online) The notch depth $\left(w_{1}\right)$ dependence of depinning field $\left(h_{c}\right)$ of a head-to-head transverse DW of anticlockwise chirality in a 64-nm-wide nanowire with a 40-nm-wide rectangular notch. The black crosses are the simulation results with the magnetostatic interaction, and the black curve is the corresponding theoretical prediction. The red squares are the simulations without the magnetostatic interaction, and the red curve is the corresponding theoretical prediction.

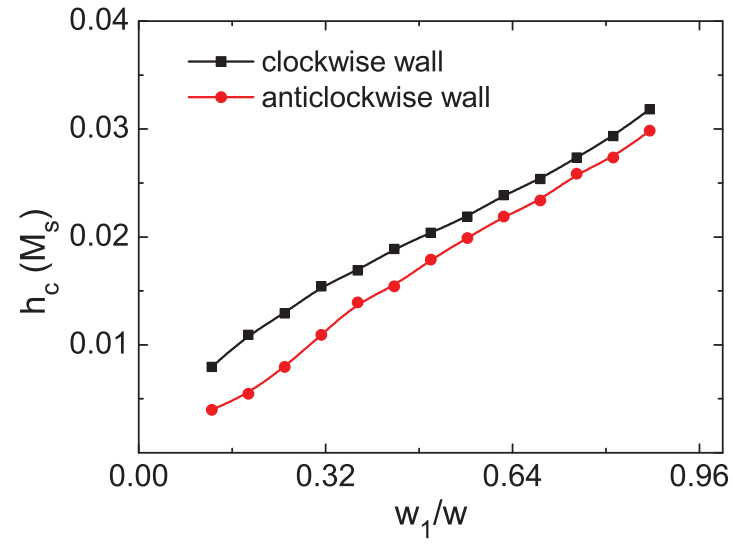

FIG. 5. (Color online) The notch depth $\left(w_{1}\right)$ dependence of the depinning field $\left(h_{c}\right)$ of head-to-head transverse DWs in a 64-nm-wide nanowire with a 40-nm-wide rectangular notch. The black squares and red dots are for the DW of clockwise and anticlockwise chirality, respectively.

$h_{c}=0$ when a notch does not exist, i.e., $w_{1}=0$, and there is no DW pinning, a well-known result [29]. The other is $w_{1} \approx w$, where a wire consists of detached parts. The spin configuration on the right-hand side of $E F$ is close to a single-domain state, and the depinning field is expected to be close to the switching fields of a single domain. The limit of the depinning field is about $450 \mathrm{Oe}$, calculating from Eq. (12) when $w_{1} \rightarrow w$, which is in good agreement with a reported value with similar parameters [36]. Our notch depth dependence of the depinning field is different from Bogart et al.'s argument on a triangular notch where a further increase of the aspect ratio above $60 \%$ has no effect on the depinning fields [12]. There sufficiently large fields will nucleate a DW on one side of the notch, while another DW is still pinned on the other side of the notch. Here only one DW pinned at the right-hand side of the notch exists under an applied field.

Simulations on a transverse wall of clockwise chirality at fixed $w=64 \mathrm{~nm}$ show a larger depinning field than that of an anticlockwise transverse wall, as shown in Fig. 5. The same behavior was found in experiments [11]. This chirality dependence of the depinning field can also be well explained by our theory. It is known that the DW width is not uniform in the transverse direction due to magnetostatic interaction $[27,32]$. The anticlockwise wall is wider at the top edge and narrower at the bottom edge, which is reversed for a clockwise wall. Therefore the anticlockwise wall whose width is larger near the notch should correspond to smaller depinning fields, as can be seen from Eq. (11).

For comparison, we also study DW depinning when the magnetostatic interaction is excluded. It should be pointed out that a DW prefers to be pinned at the notch center at zero field in this case [6]. The red squares in Fig. 4 are the notch depth dependence of the depinning field from simulations, and the red curve is the corresponding theoretical prediction. One can see that they perfectly agree with each other. This good agreement suggests that our theory captures the essential physics of DW pinning. A better fit between theory and simulations in the absence of magnetostatic interaction indicates that the main error in our prediction comes from 
the calculation of $T_{2}$, which is related to the magnetostatic interaction of the inhomogeneous DW texture.

\section{CONCLUSION}

In conclusion, an identity is derived from the EulerLagrange equation. This identity provides a relationship between an external field and a static DW structure in a notched nanowire. The depinning field corresponds to the maximal external field under which this identity holds. This theoretical estimate of the depinning field agrees well with simulation results. It also explains why the depinning field depends on the DW chirality.

\section{ACKNOWLEDGMENTS}

H.Y.Y. acknowledges the support of a Hong Kong Ph.D. Fellowship. He would also like to thank X. Wang, Y. Zhang, C. Wang, and A. Zhe for helpful discussions. This work was supported by Hong Kong GRF Grant No. 605413 and NNSF of China Grant No. 11374249.
[1] S. S. P. Parkin, M. Hayashi, and L. Thomas, Science 320, 190 (2008).

[2] D. A. Allwood, G. Xiong, C. C. Faulkner, D. Atkinson, D. Petit, and R. P. Cowburn, Science 309, 1688 (2005).

[3] D. Sander, R. Skomski, C. Schmidthals, A. Enders, and J. Kirschner, Phys. Rev. Lett. 77, 2566 (1996).

[4] P. Bruno, Phys. Rev. Lett. 83, 2425 (1999).

[5] R. D. McMichael, J. Eicke, M. J. Donahue, and D. G. Porter, J. Appl. Phys. 87, 7058 (2000).

[6] N. García, V. V. Osipov, and E. V. Ponizovskaya, Phys. Rev. B 64, 184412 (2001).

[7] V. A. Molyneux, V. V. Osipov, and E. V. Ponizovskaya, Phys. Rev. B 65, 184425 (2002).

[8] M. Kläui, C. A. F. Vaz, J. Rothman, J. A. C. Bland, W. Wernsdorfer, G. Faini, and E. Cambril, Phys. Rev. Lett. 90, 097202 (2003).

[9] A. Himeno, T. Ono, S. Nasu, K. Shigeto, K. Mibu, and T. Shinjo, J. Appl. Phys. 93, 8430 (2003).

[10] M. Kläui, H. Ehrke, U. Rüdiger, T. Kasama, R. E. Dunin-Borkowski, D. Backes, L. J. Heyderman, C. A. F. Vaz, J. A. C. Bland, G. Faini, E. Cambril, and W. Wernsdorfer, Appl. Phys. Lett. 87, 102509 (2005).

[11] M. Hayashi, L. Thomas, C. Rettner, R. Moriya, X. Jiang, and S. S. P. Parkin, Phys. Rev. Lett. 97, 207205 (2006).

[12] L. K. Bogart, D. S. Eastwood, and D. Atkinson, J. Appl. Phys. 104, 033904 (2008).

[13] C. C. Faulkner, D. A. Allwood, and R. P. Cowburn, J. Appl. Phys. 103, 073914 (2008).

[14] S. Lepadatu, A. Vanhaverbeke, D. Atkinson, R. Allenspach, and C. H. Marrows, Phys. Rev. Lett. 102, 127203 (2009).

[15] L. K. Bogart, D. Atkinson, K. O'Shea, D. McGrouther, and S. McVitie, Phys. Rev. B 79, 054414 (2009).

[16] M.-Y. Im, L. Bocklage, P. Fischer, and G. Meier, Phys. Rev. Lett. 102, 147204 (2009).

[17] D. Petit, A. V. Jausovec, H. T. Zeng, E. Lewis, L. O’Brien, D. Read, and R. P. Cowburn, Phys. Rev. B 79, 214405 (2009).
[18] A. Kunz and J. D. Priem, IEEE Trans. Magn. 46, 1559 (2010).

[19] D. Djuhana, H.-G. Piao, S.-H. Lee, D.-H. Kim, S.-M. Ahn, and S.-B. Choe, Appl. Phys. Lett. 97, 022511 (2010).

[20] F. Garcia-Sanchez, A. Kákay, R. Hertel, and P. Asselin, Appl. Phys. Express 4, 033001 (2011).

[21] U.-H. Pi, Y.-J. Cho, J.-Y. Bae, S.-C. Lee, S. Seo, W. Kim, J.-H. Moon, K.-J. Lee, and H.-W. Lee, Phys. Rev. B 84, 024426 (2011).

[22] S. Glathe and R. Mattheis, Phys. Rev. B 85, 024405 (2012).

[23] A. Bisig, M. Stärk, M.-A. Mawass, C. Moutafis, J. Rhensius, J. Heidler, F. Büttner, M. Noske, M. Weigand, S. Eisebitt, T. Tyliszczak, B. V. Waeyenberge, H. Stoll, G. Schütz, and M. Kläui, Nat. Commun. 4, 2328 (2013).

[24] S.-M. Ahn, K.-W. Moon, D.-H. Kim, and S.-B. Choe, J. Appl. Phys. 111, 07D309 (2012).

[25] X. S. Wang, P. Yan, Y. H. Shen, G. E. W. Bauer, and X. R. Wang, Phys. Rev. Lett. 109, 167209 (2012).

[26] B. Hu and X. R. Wang, Phys. Rev. Lett. 111, 027205 (2013).

[27] Y. Nakatani, A. Thiaville, and J. Miltat, J. Magn. Magn. Mater. 290-291, 750 (2005).

[28] M. Morini and V. Slastikov, Arch. Ration. Mech. Anal. 203, 621 (2012).

[29] X. R. Wang, P. Yan, J. Lu, and C. He, Ann. Phys. (NY) 324, 1815 (2009); X. R. Wang, P. Yan, and J. Lu, Europhys. Lett. 86, 67001 (2009).

[30] A. Aharoni, J. Appl. Phys. 83, 3432 (1998).

[31] D. G. Porter and M. J. Donahue, J. Appl. Phys. 95, 6729 (2004).

[32] H. Y. Yuan and X. R. Wang, arXiv:1307.7269.

[33] R. D. McMichael and M. J. Donahue, IEEE Trans. Magn. 33, 4167 (1997).

[34] http://math.nist.gov/oommf.

[35] L. Thomas, C. Rettner, M. Hayashi, M. G. Samant, S. S. P. Parkin, A. Doran, and A. Scholl, Appl. Phys. Lett. 87, 262501 (2005).

[36] J. Lu and X. R. Wang, J. Magn. Magn. Mater. 321, 2916 (2009). 